\title{
Exchange-correlation hole of a generalized gradient approximation for solids and surfaces
}

\author{
Lucian A. Constantin, ${ }^{1}$ John P. Perdew, ${ }^{1}$ and J. M. Pitarke ${ }^{2,3}$ \\ ${ }^{1}$ Department of Physics and Quantum Theory Group, Tulane University, New Orleans, Louisiana 70118, USA \\ ${ }^{2}$ CIC nanoGUNE Consolider, Mikeletegi Pasealekua 56, E-20009 Donostia, Basque Country, Spain \\ ${ }^{3}$ Materia Kondentsatuaren Fisika Saila (UPV/EHU), DIPC, and Centro Física Materiales CSIC-UPV/EHU, 644 Posta kutxatila, \\ E-48080 Bilbo, Basque Country, Spain
}

(Received 27 October 2008; published 27 February 2009)

\begin{abstract}
We propose a generalized gradient approximation (GGA) for the angle- and system-averaged exchangecorrelation hole of a many-electron system. This hole, which satisfies known exact constraints, recovers the Perdew-Burke-Ernzerhof for solids (PBEsol) exchange-correlation energy functional, a GGA that accurately describes the equilibrium properties of densely packed solids and their surfaces. We find that our PBEsol exchange-correlation hole describes the wave-vector analysis of the jellium exchange-correlation surface energy in agreement with a sophisticated time-dependent density-functional calculation (whose three-dimensional wave-vector analysis we report here).
\end{abstract}

DOI: 10.1103/PhysRevB.79.075126

PACS number(s): 71.10.Ca, 71.15.Mb, 71.45.Gm

\section{INTRODUCTION}

In the Kohn-Sham (KS) density-functional theory ${ }^{1}$ for the ground-state energy of a many-electron system, only the exchange-correlation (xc) energy $E_{\mathrm{xc}}[n]$ has to be approximated. The exact xc energy of an arbitrary inhomogeneous system of density $n(\mathbf{r})$, which incorporates all the quantum many-body effects beyond the Hartree approximation, can be obtained from the spherical average $\bar{n}_{\mathrm{xc}}(\mathbf{r}, u)$ of the couplingconstant-averaged xc-hole density $\bar{n}_{\mathrm{xc}}\left(\mathbf{r}, \mathbf{r}^{\prime}\right)$ at $\mathbf{r}^{\prime}$ around an electron at $\mathbf{r}$ as follows: $:^{2,3}$

$$
E_{\mathrm{xc}}[n]=\int d \mathbf{r} n(\mathbf{r}) \varepsilon_{\mathrm{xc}}[n](\mathbf{r}),
$$

where $\varepsilon_{\mathrm{xc}}[n](\mathbf{r})$ is the $\mathrm{xc}$ energy per particle at point $\mathbf{r}$,

$$
\begin{aligned}
\varepsilon_{\mathrm{xc}}[n](\mathbf{r}) & =\frac{1}{2} \int_{0}^{\infty} d u 4 \pi u^{2} \frac{1}{u} \bar{n}_{\mathrm{xc}}(\mathbf{r}, u) \\
& =4 \int_{0}^{\infty} d k \int_{0}^{\infty} d u u^{2} \frac{\sin k u}{k u} \bar{n}_{\mathrm{xc}}(\mathbf{r}, u),
\end{aligned}
$$

with

$$
\bar{n}_{\mathrm{xc}}(\mathbf{r}, u)=\frac{1}{4 \pi} \int d \Omega \bar{n}_{\mathrm{xc}}\left(\mathbf{r}, \mathbf{r}^{\prime}\right),
$$

with $d \Omega$ being a differential solid angle around the direction of $\mathbf{u}=\mathbf{r}^{\prime}-\mathbf{r}$ and $k$ representing the magnitude of the wave vector. (Unless otherwise stated, atomic units are used throughout, i.e., $e^{2}=\hbar=m_{e}=1$.)

The "Jacob's ladder" classification of the widely used ground-state density-functional approximations for $E_{\mathrm{xc}}[n]$ and $\bar{n}_{\mathrm{xc}}(\mathbf{r}, u)$ has three complete nonempirical rungs: the local spin-density approximation (LSDA), ${ }^{1}$ the generalized gradient approximation (GGA), ${ }^{4-6}$ and the meta-GGA. ${ }^{7,8}$ Due to its simplicity and accuracy, one of the most commonly used xc density-functional approximation in solidstate physics and quantum chemistry calculations is nowadays the semilocal Perdew-Burke-Ernzerhof (PBE) GGA. ${ }^{4}$
Recent work ${ }^{9}$ has shown, however, that the exchange density-functional approximations should recover, in the limit of slowly varying densities, the universal second-order gradient-expansion (GE) approximation of the exchange energy,

$$
E_{x}^{\mathrm{GE}}[n]=\int d \mathbf{r} n(\mathbf{r}) \epsilon_{x}^{\mathrm{unif}}(n(\mathbf{r}))\left[1+\mu_{x}^{\mathrm{GE}} s^{2}(\mathbf{r})+\cdots\right],
$$

where $\epsilon_{x}^{\text {unif }}$ is the exchange energy per particle of the uniform electron gas, $\mu_{x}^{\mathrm{GE}}=10 / 81$ is the GE exchange coefficient, ${ }^{10}$ and $s=|\nabla n| /\left(2 k_{F} n\right)$ is the reduced density gradient which measures the variation in the electron density over a Fermi wavelength $\lambda_{F}=2 \pi / k_{F}$, with $k_{F}=\left(3 \pi^{2} n\right)^{1 / 3}$ representing the magnitude of the local Fermi wave vector. Recovery of the correct second-order gradient expansion for correlation ${ }^{11}$ in the slowly varying limit is much less important for the construction of density-functional approximations. (See Table I of Ref. 9.)

A GGA, which has as ingredients only the spin densities $n_{\uparrow}$ and $n_{\downarrow}$ and their gradients $\nabla n_{\uparrow}$ and $\nabla n_{\downarrow}$, cannot recover, in the limit of slowly varying densities, the GE approximation of the exchange energy and at the same time be accurate for atoms. ${ }^{9,12}$ The semilocal PBE has the correct correlation GE coefficient in the high-density limit and is accurate for atoms, but its exchange GE coefficient is almost twice as large as the exact coefficient, i.e., $\mu_{x}^{\mathrm{PBE}} \approx 2 \mu_{x}^{\mathrm{GE}}$. Because of this, ${ }^{12}$ PBE overestimates the equilibrium lattice constants of solids and yields surface energies that are too low.

Following the ideas of Ref. 9, PBE for solids (PBEsol) was constructed. ${ }^{12}$ PBEsol is a GGA that has the same form as $\mathrm{PBE}$ but restores the density-gradient expansion for exchange by replacing $\mu_{x}^{\mathrm{PBE}}=0.2195$ with $\mu_{x}^{\mathrm{PBEsol}}=\mu_{x}^{\mathrm{GE}}$. By fitting the jellium xc surface energies (as done previously in Ref. 13 in the construction of a GGA which relies on the Airy-gas approximation ${ }^{14}$ ), the PBEsol correlation GE coefficient was set to $\mu_{c}^{\mathrm{PBEsol}}=0.046$. (For PBE, $\mu_{c}^{\mathrm{PBE}}=0.0667$.) Thus, PBEsol can easily be applied in solid-state calculations (just by changing the coefficients in a PBE code) and yields good equilibrium lattice constants and jellium surface 
energies. ${ }^{12}$ Several other applications of PBEsol have already proven the accuracy of this GGA for solids. In particular, PBEsol considerably improves the structure of gold clusters $^{15}$ and works better than PBE for isomerization energies and isodesmic stabilization energies of hydrocarbon molecules. ${ }^{16}$ PBEsol also describes ferroelectric and antiferroelectric $\mathrm{ABO}_{3}$ crystals $^{17}$ much better than LSDA or PBE GGA.

In this paper, we first construct the PBEsol angleaveraged xc-hole density $\bar{n}_{\mathrm{xc}}^{\mathrm{PBEs}}(\mathbf{r}, u)$. A nonempirical derivation of the PBE xc hole was reported in Ref. 5, starting from the second-order density-gradient expansion of the $\mathrm{xc}$ hole and cutting off the spurious large- $u$ contributions to satisfy exact constraints, according to which (i) the exchangehole density must be negative, (ii) the exchange hole must integrate to -1 , and (iii) the correlation hole must integrate to zero. Later on, a fully smoothed analytic model was constructed for the PBE exchange hole. ${ }^{6}$ Our construction of the PBEsol xc hole begins with and appropriately modifies the sharp cutoff correlation hole of Ref. 5 and the smooth $e x$ change hole of Ref. 6 . It should be recalled that, because of an integration by parts that occurs in the underlying gradient expansion, a GGA hole is meaningful only after averaging over the electron density $n(\mathbf{r})$ (as in our tests and applications), and this system averaging itself smoothes sharp cutoffs.

Finally, we use our PBEsol xc hole to carry out a threedimensional (3D) wave-vector analysis of the jellium xc surface energy. This wave-vector analysis was carried out in Ref. 3 in the random-phase approximation (RPA). Here, we go beyond the RPA in the framework of time-dependent density-functional theory (TDDFT), and we compare these calculations with the results we obtain from our PBEsol xchole density.

This paper is organized as follows. In Sec. II, we present the PBEsol angle-averaged xc-hole density $\bar{n}_{\mathrm{xc}}^{\mathrm{PBEs}}(\mathbf{r}, u)$. In Sec. III, we perform the wave-vector analysis of the jellium xc surface energy. In Sec. IV, we summarize our conclusions.

\section{PBEsol-GGA ANGLE-AVERAGED EXCHANGE- CORRELATION HOLE}

We assume here that the PBEsol correlation energy can be constructed from a gradient expansion for the correlation hole in much the same way that the PBE correlation energy was so constructed. ${ }^{5}$ The GGA angle-averaged correlation hole is 5

$$
\bar{n}_{c}^{\mathrm{GGA}}\left(r_{s}, \zeta, t, v\right)=\phi^{5} k_{s}^{2}\left[A_{c}\left(r_{s}, \zeta, v\right)+t^{2} B_{c}\left(r_{s}, \zeta, v\right)\right] \theta\left(v_{c}-v\right),
$$

where $r_{s}=(9 \pi / 4)^{1 / 3} / k_{F}$ is a local-density parameter, $\zeta=\left(n_{\uparrow}\right.$ $\left.-n_{\downarrow}\right) /\left(n_{\uparrow}+n_{\downarrow}\right)$ is the relative spin polarization, $\phi=\left[(1+\zeta)^{2 / 3}\right.$ $\left.+(1-\zeta)^{2 / 3}\right] / 2$ is a spin-scaling factor, $v=\phi k_{s} u$ with $k_{s}$ $=\left(4 k_{F} / \pi\right)^{1 / 2}$ is the reduced electron-electron separation on the scale of the screening length, and $t=|\nabla n| /\left(2 \phi k_{s} n\right)$ is the reduced density gradient measuring the variation in the electron density over the screening length. The sharp cutoff $v_{c}$ is found such that Eq. (5) satisfies the correlation hole sum rule

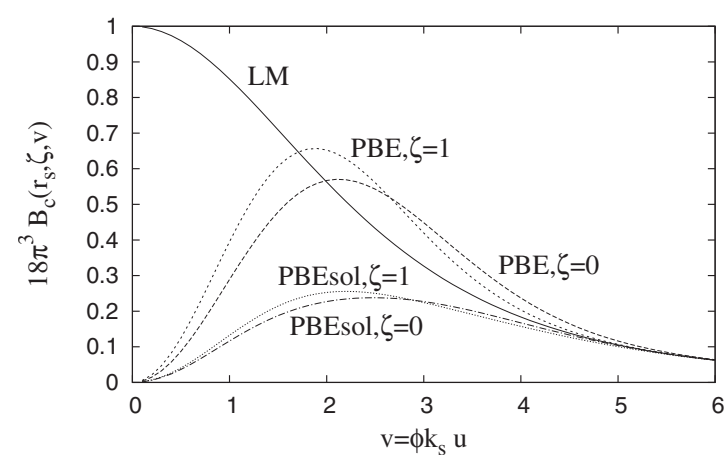

FIG. 1. Gradient correction to the correlation hole, $B_{c}\left(r_{s}, \zeta, v\right)$, versus $v$ for $r_{s}=2$. PBE and PBEsol are compared here. The solid line represents the Langreth-Mehl (LM) RPA contribution, which should be recovered at large $v$.

$\int d \mathbf{r} n_{c}\left(\mathbf{r}, \mathbf{r}^{\prime}\right)=0 . \quad \phi^{5} k_{s}^{2} A_{c}\left(r_{s}, \zeta, v\right)$ is the LSDA correlation hole ${ }^{18}$ given by Eq. (45) of Ref. 5, and the gradient correction to the correlation hole is given by the following expression: ${ }^{5}$

$$
B_{c}\left(r_{s}, \zeta, v\right)=B_{c}^{\mathrm{LM}}(v)\left[1-e^{-p v^{2}}\right]+\beta\left(r_{s}, \zeta\right) v^{2} e^{-p v^{2}},
$$

where $B_{c}^{\mathrm{LM}}(v)$ is the RPA nonoscillating long-range contribution given by Eq. (49) of Ref. 5, $p\left(r_{s}, \zeta\right)=\pi k_{F}(0.305$ $\left.-0.136 \zeta^{2}\right) / 4 \phi^{4}$ measures where the short-range contribution vanishes, and

$$
\beta\left(r_{s}, \zeta\right)=\frac{2 p^{2}}{3 \pi^{3}}\left[\frac{\mu_{c}^{\mathrm{GGA}}}{\mu_{c}^{\mathrm{PBE}}}-E_{1}(12 p)\right]
$$

is constructed so that the second-order gradient expansion of the PBEsol correlation energy is recovered. Here $E_{1}(y)$ $=y e^{y} \int_{y}^{\infty} d t e^{-t} / t$ is between 0 and 1 , and $\mu_{c}^{\mathrm{GGA}}$ is the GGA gradient coefficient in the slowly varying limit $\left(\mu_{c}^{\mathrm{PBE}}\right.$ for PBE and $\mu_{c}^{\mathrm{PBEsol}}$ for PBEsol).

In Fig. 1, we show PBE and PBEsol versions of $B_{c}\left(r_{s}, \zeta, v\right)$ versus $v$ for $r_{s}=2$. Both PBE and PBEsol recover the correct RPA-like behavior $\left[B_{c}^{\mathrm{LM}}(v)\right]$ at large $v$, and they both show the same $\zeta$ behavior; because $\mu_{c}^{\mathrm{PBEsol}}<\mu_{c}^{\mathrm{PBE}}$, however, at intermediate values of $v$ the PBEsol gradient correction to the correlation hole is substantially smaller than the PBE one. The gradient correction $B_{c}\left(r_{s}, \zeta, v\right)$ of Eq. (6) can be negative at small $v$ and small $r_{s}$; however, because of the energy sum rule both $\int_{0}^{\infty} d u u B_{c}\left(r_{s}, \zeta, v\right)$ and $\int_{0}^{\infty} d u u^{2} B_{c}\left(r_{s}, \zeta, v\right)$ are positive, which ensures that the cutoff procedure is correct and for every value of $r_{s}, \zeta$, and $t$ there is a $v_{c}$ such that Eq. (5) satisfies the correlation-hole sum rule.

The exchange energy and exchange-hole density for a spin-polarized system may be evaluated from their spinunpolarized counterparts by using the spin-scaling relations ${ }^{5,19}$

$$
E_{x}\left[n_{\uparrow}, n_{\downarrow}\right]=\frac{1}{2}\left\{E_{x}\left[2 n_{\uparrow}\right]+E_{x}\left[2 n_{\downarrow}\right]\right\}
$$




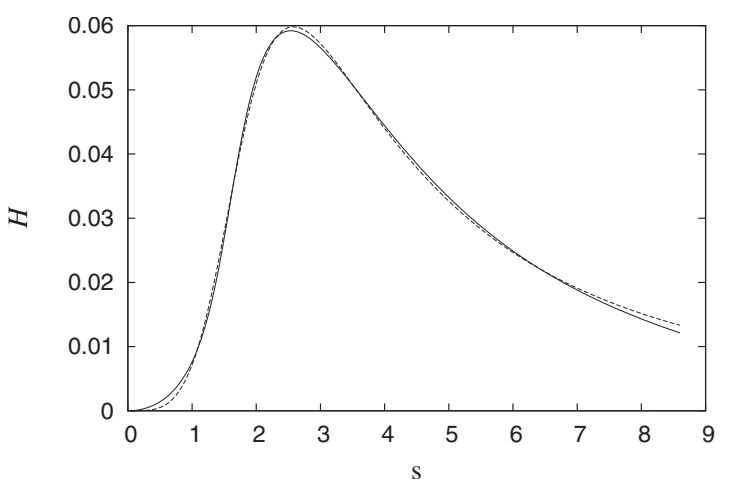

FIG. 2. The exponent $\mathcal{H}(s)$ of Eq. (10) versus the reduced gradient $s$. The solid line represents the numerical solution of the implicit equation for $\mathcal{H}(s)$ [Eq. (A4) of Ref. 6]. The dashed line represents the fit of Eq. (14).

$$
n_{x}\left[n_{\uparrow}, n_{\downarrow}\right](\mathbf{r}, \mathbf{r}+\mathbf{u})=\sum_{\sigma} \frac{n_{\sigma}(\mathbf{r})}{n(\mathbf{r})} n_{x}\left[2 n_{\sigma}\right](\mathbf{r}, \mathbf{r}+\mathbf{u})
$$

thus, we only need to consider the spin-unpolarized system. As in the case of the analytical PBE exchange hole of Ref. 6, we choose the following ansatz for the nonoscillatory dimensionless exchange-hole shape:

$$
\begin{aligned}
J^{\mathrm{PBEsol}}(s, y)= & \left\{-\frac{\mathcal{A}}{y^{2}} \frac{1}{1+(4 / 9) \mathcal{A} y^{2}}+\left[\frac{\mathcal{A}}{y^{2}}+\mathcal{B}+\mathcal{C}(1\right.\right. \\
& \left.\left.\left.+s^{2} \mathcal{F}(s)\right) y^{2}+\mathcal{E}\left(1+s^{2} \mathcal{G}(s)\right) y^{4}\right] e^{-\mathcal{D} y^{2}}\right\} e^{-s^{2} \mathcal{H}(s) y^{2}},
\end{aligned}
$$

where $s$ is the reduced density gradient for exchange. When $s=0$, Eq. (10) recovers ${ }^{6,20} J^{\mathrm{LSDA}}$ for $\mathcal{A}=1.0161144, \mathcal{B}$ $=-0.37170836, \mathcal{C}=-0.077215461, \mathcal{D}=0.57786348$, and $\mathcal{E}=-0.051955731$. The functions $\mathcal{F}(s), \mathcal{G}(s)$, and $\mathcal{H}(s)$ are found in such a way that the energy and exchange-hole sum rules are satisfied,

$$
\frac{8}{9} \int_{0}^{\infty} d y y J^{\mathrm{PBEsol}}(s, y)=-F_{x}^{\mathrm{PBEsol}}(s)
$$

and

$$
\frac{4}{3 \pi} \int_{0}^{\infty} d y y^{2} J^{\mathrm{PBEsol}}(s, y)=-1,
$$

and also the small- $u$ behavior of the exchange hole is recovered by ${ }^{6}$

$$
\mathcal{F}(s)=6.475 \mathcal{H}(s)+0.4797 .
$$

Here $F_{x}^{\mathrm{PBEsol}}(s)$ is the PBEsol enhancement factor. ${ }^{12}$ The integrals of Eqs. (11) and (12) can be solved analytically, ${ }^{6}$ and Eqs. (11)-(13) reduce (by substitution) to an implicit equation for $\mathcal{H}$ [Eq. (A4) of Ref. 6]. We have solved this equation for PBEsol; the numerical solution that we have found for $\mathcal{H}(s)$ (see Fig. 2) can be fitted to the following analytic expression:

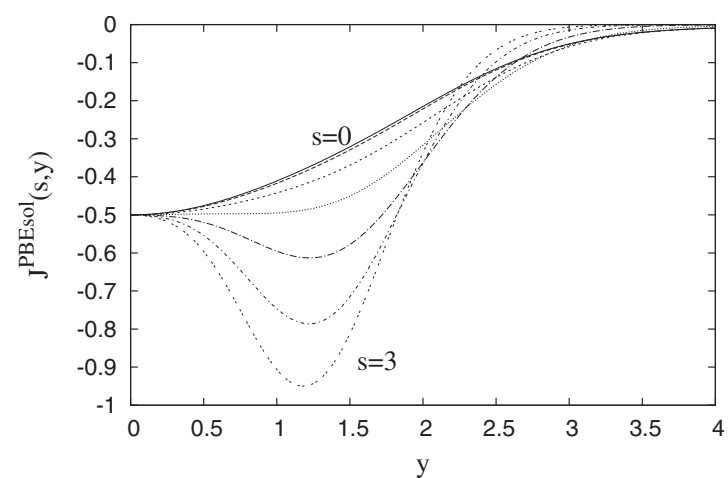

FIG. 3. Dimensionless exchange-hole shape $J^{\mathrm{PBEsol}}(s, y)$ [see Eq. (10)] versus $y=k_{F} u$ for $s$ between 0 and 3 in steps of 0.5. For comparison with $J^{\mathrm{PBE}}(s, y)$, see Fig. 2 of Ref. 6. When $s=0$, $J^{\mathrm{PBEsol}}(s, y)$ yields $J^{\mathrm{LSDA}}(y)$.

$$
\mathcal{H}(s)=\frac{a_{1} s^{2}+a_{2} s^{4}}{1+a_{3} s^{4}+a_{4} s^{6}},
$$

where $\quad a_{1}=0.00018855, \quad a_{2}=0.00741358, \quad a_{3}$ $=0.05687256$, and $a_{4}=0.00675093$. For $s>8.5$, as occurs in the tail of an atom or molecule where the electron density is negligible, the implicit equation for $\mathcal{H}(s)$ does not have a solution (as in the PBE case ${ }^{6}$ ) so we reset $s$ to $s=8.5$. Recently, Henderson et al. ${ }^{21}$ constructed a GGA exchange hole that eliminates this unphysical large- $s$ behavior by using some ideas from the meta-GGA hole. ${ }^{7}$

In Fig. 3, we plot the dimensionless exchange-hole shape $J^{\text {PBEsol }}(s, y)$ [using the analytical fit of Eq. (14)] versus $y$ $=k_{F} u$ for several values of the reduced gradient $s$. Our $J^{\mathrm{PBEsol}}(s, y)$ looks similar to the $J^{\mathrm{PBE}}(s, y)$ of Ref. 6 , but $J^{\mathrm{PBE}}(s, y)$ is deeper because $\mu_{x}^{\mathrm{PBE}}=0.2195>\mu_{x}^{\mathrm{PBEsol}}=0.1235$.

Finally, we look at the xc enhancement factor, which displays the nonlocality, ${ }^{22}$

$$
F_{\mathrm{xc}}^{\mathrm{GGA}}=\frac{\epsilon_{\mathrm{xc}}^{\mathrm{GGA}}\left(n_{\uparrow}, n_{\downarrow}, \nabla n_{\uparrow}, \nabla n_{\downarrow}\right)}{\epsilon_{x}^{\mathrm{unif}}(n)},
$$

with $\epsilon_{x}^{\text {unif }}(n)$ being the exchange energy per particle of a spinunpolarized uniform electron gas. For a spin-unpolarized system in the high-density limit $\left(r_{s} \rightarrow 0\right)$ the exchange energy is dominant and Eq. (15) defines the exchange enhancement factor $F_{x}^{\mathrm{GGA}}=\epsilon_{x}^{\mathrm{GGA}}(n, \nabla n) / \epsilon_{x}^{\mathrm{unif}}(n)$. In Figs. 4 and 5, we show the PBEsol enhancement factor for a spin-unpolarized system, $F_{\mathrm{xc}}^{\mathrm{PBEsol}}\left(r_{s}, \zeta=0, s\right)$, and for a fully spin-polarized system, $F_{\mathrm{xc}}^{\mathrm{PBEsol}}\left(r_{s}, \zeta=1, s\right)$, versus $s$ for several values of $r_{s}$. $F_{\mathrm{xC}}^{\mathrm{PB}} \mathrm{Xol}$ is calculated either (i) from the analytic expression of $\epsilon_{\mathrm{xc}}^{\mathrm{PBEs}}$ reported in Ref. 12 or (ii) from our PBEsol angleaveraged xc-hole density through Eq. (2). Overall, these calculations of $F_{\text {xc }}^{\text {PBEsol }}$ agree well with each other, confirming the assumption made at the beginning of this section; only for $r_{s} \geq 10$ (when the electron density is very small) and $s$ $\approx 1.5$ is the error introduced by the second procedure significant. ${ }^{23}$ The analytic fit for $\mathcal{H}(s)$ used to construct our PBEsol exchange hole does not exactly reproduce the PBEsol enhancement factor, but the difference is small as shown in Fig. 4. At this point, we also note that the parametrization ${ }^{4}$ of $H\left(r_{s}, \zeta, t\right)$ entering the analytic expression of $\epsilon_{c}^{\text {PBEsol }}$ re- 


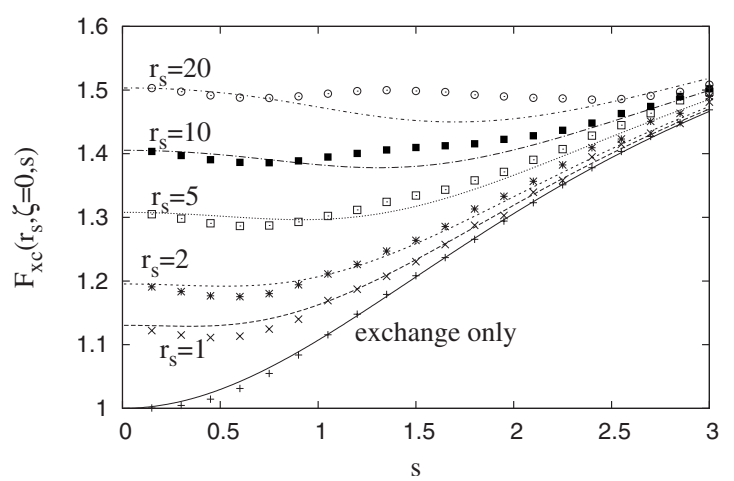

FIG. 4. The PBEsol enhancement factor $F_{\mathrm{xc}}$ for the spinunpolarized case $(\zeta=0)$ as a function of the reduced gradient $s$ for several values of $r_{s}$. The lines represent the enhancement factor obtained from the PBEsol xc energy functional of Ref. 12. The dots represent the enhancement factor obtained from our PBEsol angleaveraged xc-hole density through Eq. (2).

ported in Ref. 4 does not reproduce exactly the real-space cutoff results, as shown in Figs. 7 and 8 of Ref. 5.

\section{WAVE-VECTOR ANALYSIS OF THE JELLIUM XC SURFACE ENERGY}

The xc surface energy, $\sigma_{\mathrm{xc}}$, can be defined as the xc energy cost per unit area to create a planar surface by cutting the bulk. In a jellium model, in which the electron system is translationally invariant in the plane of the surface, and assuming the surface to be normal to the $z$ axis, the surface energy can be written as follows: ${ }^{3}$

$$
\sigma_{\mathrm{xc}}=\int_{0}^{\infty} d\left(\frac{k}{2 k_{F}}\right) \gamma_{\mathrm{xc}}(k),
$$

where $^{24}$

$$
\gamma_{\mathrm{xc}}(k)=2 \frac{k_{F}}{\pi} \int_{-\infty}^{+\infty} d z n(z) b_{\mathrm{xc}}(k, z)
$$

is the wave-vector-resolved xc surface energy, and

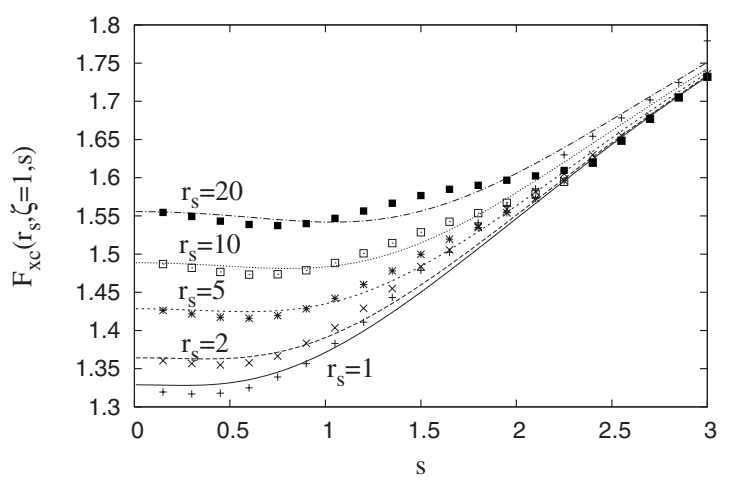

FIG. 5. The PBEsol enhancement factor $F_{\mathrm{xc}}$ for the fully spinpolarized case $(\zeta=1)$ as a function of the reduced gradient $s$ for several values of $r_{s}$. The lines represent the enhancement factor obtained from the PBEsol xc energy functional of Ref. 12. The dots represent the enhancement factor obtained from our PBEsol angleaveraged xc-hole density through Eq. (2).

$$
b_{\mathrm{xc}}(k, z)=4 \pi \int_{0}^{\infty} d u u^{2} \frac{\sin k u}{k u}\left[\bar{n}_{\mathrm{xc}}(z, u)-\bar{n}_{\mathrm{xc}}^{\mathrm{unif}}(u)\right] .
$$

Equations (16)-(18) comprise an angle-averaged threedimensional wave-vector analysis of the surface xc energy into contributions from density fluctuations of various wave vectors $k$, following from the Fourier transform of the Coulomb interaction in Eq. (2). In these and subsequent equations, $k_{F}=\left(3 \pi^{2} \bar{n}\right)^{1 / 3}$ is the bulk (not the local) Fermi wave vector and $r_{s}$ is the bulk (not the local) density parameter.

The exact low wave-vector limit of $\sigma_{\mathrm{xc}}$ is known to be ${ }^{2}$

$$
\gamma_{\mathrm{xc}}(k \rightarrow 0)=\frac{k_{F}}{4 \pi}\left(\omega_{s}-\frac{1}{2} \omega_{p}\right) k
$$

where $\omega_{p}=(4 \pi \bar{n})^{1 / 2}$ and $\omega_{s}=\omega_{p} / \sqrt{2}$ are the bulk- and surface-plasmon energies and $\bar{n}$ is the bulk density. Equation (19) was used in the wave-vector-interpolation approach reported in Refs. 2, 7, and 25, and it was naturally recovered by the RPA approach reported in Ref. 3 .

Taking into account that $\mathbf{k} \equiv\left(\mathbf{k}_{\|}, \mathbf{k}_{\mathbf{z}}\right)$, with $\mathbf{k}_{\|}$being a wave vector parallel to the surface, Eq. (18) can be expressed as follows: ${ }^{3}$

$b_{\mathrm{xc}}(k, z)=\left[\frac{1}{2} \int_{-k}^{+k} \frac{d k_{z}}{k} \int_{-\infty}^{+\infty} d z^{\prime} e^{i k_{z}\left(z-z^{\prime}\right)} \bar{n}_{\mathrm{xc}}\left(k_{\|} ; z, z^{\prime}\right)-\bar{n}_{\mathrm{xc}}^{\mathrm{unif}}(k)\right]$.

In the case of RPA and TDDFT calculations, we use the fluctuation-dissipation theorem ${ }^{2,26,27}$ to derive $n_{\mathrm{xc}}\left(k_{\|} ; z, z^{\prime}\right)$ and $\bar{n}_{\mathrm{xc}}^{\mathrm{unif}}(k)$ from the coupling-constant-dependent densityresponse functions $\chi_{\lambda}^{\text {unif }}(k)$ and $\chi_{\lambda}\left(k_{\|} ; z, z^{\prime}\right)$ as follows: ${ }^{3,28}$

$$
\bar{n}_{\mathrm{xc}}^{\mathrm{unif}}(k)=\frac{1}{\bar{n}}\left[-\frac{1}{\pi} \int_{0}^{1} d \lambda \int_{0}^{\infty} d \omega \chi_{\lambda}^{\mathrm{unif}}(k, i \omega)-\bar{n}\right]
$$

and

$$
\begin{aligned}
\bar{n}_{\mathrm{xc}}\left(k_{\|} ; z, z^{\prime}\right)= & \frac{1}{n(z)}\left[-\frac{1}{\pi} \int_{0}^{1} d \lambda \int_{0}^{\infty} d \omega \chi_{\lambda}\left(z, z^{\prime} ; k_{\|}, i \omega\right)\right. \\
& \left.-n(z) \delta\left(z-z^{\prime}\right)\right],
\end{aligned}
$$

with $\chi_{\lambda}^{\text {unif }}(k, \omega)$ and $\chi_{\lambda}\left(z, z^{\prime} ; k_{\|}, i \omega\right)$ being the 3D and 2D Fourier transforms of the corresponding density-response function $\chi_{\lambda}\left(\mathbf{r}, \mathbf{r}^{\prime} ; \omega\right)$. In the framework of TDDFT (our benchmark for this work), the density-response function $\chi_{\lambda}\left(\mathbf{r}, \mathbf{r}^{\prime} ; \omega\right)$ satisfies a Dyson-type equation of the form ${ }^{29}$

$$
\begin{aligned}
\chi_{\lambda}\left(\mathbf{r}, \mathbf{r}^{\prime} ; \omega\right)= & \chi_{0}\left(\mathbf{r}, \mathbf{r}^{\prime} ; \omega\right)+\int d \mathbf{r}_{1} d \mathbf{r}_{2} \chi_{0}\left(\mathbf{r}, \mathbf{r}_{1} ; \omega\right)\left\{\frac{\lambda}{\left|\mathbf{r}_{1}-\mathbf{r}_{2}\right|}\right. \\
& \left.+f_{\mathrm{xc}, \lambda}[n]\left(\mathbf{r}_{1}, \mathbf{r}_{2} ; \omega\right)\right\} \chi_{\lambda}\left(\mathbf{r}_{2}, \mathbf{r}^{\prime} ; \omega\right),
\end{aligned}
$$

where $\chi_{0}\left(\mathbf{r}, \mathbf{r}^{\prime} ; \omega\right)$ is the density-response function of noninteracting $\mathrm{KS}$ electrons (which is exactly expressible in terms of KS orbitals $\left.{ }^{30}\right)$ and $f_{\mathrm{xc}, \lambda}[n]\left(\mathbf{r}, \mathbf{r}^{\prime} ; \omega\right)$ is the unknown $\lambda$-dependent dynamic xc kernel. When $f_{\mathrm{xc}, \lambda}[n]\left(\mathbf{r}, \mathbf{r}^{\prime} ; \omega\right)$ is taken to be zero, Eq. (23) reduces to the RPA density- 


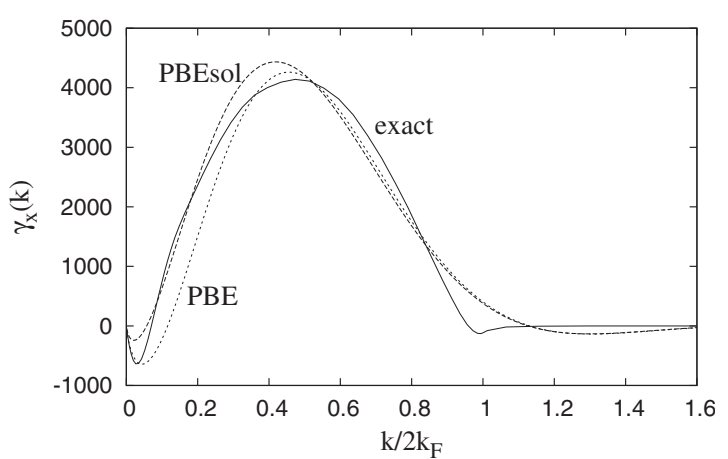

FIG. 6. PBE, PBEsol, and exact wave-vector-resolved exchange surface energies $\gamma_{x}(k)$ versus $k / 2 k_{F}$ for a jellium slab of thickness $a=2.23 \lambda_{F}$ and $r_{s}=2.07$. The semilocal PBE and PBEsol calculations have been performed from nonoscillatory parametrizations of the dimensionless exchange-hole shapes $J^{\mathrm{PBE}}$ and $J^{\mathrm{PBEsol}}$, respectively. The area under each curve represents the corresponding exchange surface energy: $\sigma_{x}^{\mathrm{PBE}}=2164 \mathrm{erg} / \mathrm{cm}^{2}, \quad \sigma_{x}^{\mathrm{PBEs} o l}$ $=2424 \mathrm{erg} / \mathrm{cm}^{2}$, and $\sigma_{x}^{\text {exact }}=2348 \mathrm{erg} / \mathrm{cm}^{2}$.

response function. If the interacting density-response function $\chi_{\lambda}\left(\mathbf{r}, \mathbf{r}^{\prime} ; \omega\right)$ is replaced by the noninteracting $\mathrm{KS}$ density-response function $\chi_{0}\left(\mathbf{r}, \mathbf{r}^{\prime} ; \omega\right)$, then Eqs. (21) and (22) yield their exchange-only counterparts.

In the calculations presented below, we have considered, as in Ref. 3, a jellium slab of background thickness $a$ $=2.23 \lambda_{F}$ (where $\left.\lambda_{F}=2 \pi / k_{F}\right)$ and bulk parameter $r_{s}=2.07$. This slab corresponds to about four atomic layers of $\mathrm{Al}(100)$.

For the GGA calculations of $\gamma_{\mathrm{xc}}(k)$, the function $b_{\mathrm{xc}}(k, z)$ entering Eq. (17) is taken from Eq. (18) with the xc-hole densities calculated as reported in (i) Ref. 20 for $\bar{n}_{\mathrm{xc}}^{\text {unif }}(u)$, (ii) Refs. 5 and 6 for $\bar{n}_{\mathrm{xc}}^{\mathrm{PBE}}(z, u)$, and (iii) Sec. II above for $\bar{n}_{\mathrm{xc}}^{\mathrm{PBEsol}}(z, u)$.

For the exact-exchange, exact-RPA, and TDDFT calculations of $\gamma_{\mathrm{xc}}(k)$, the function $b_{\mathrm{xc}}(k, z)$ entering Eq. (17) is taken from Eq. (20) with the xc-hole densities calculated from Eqs. (21) and (22). In the case of the TDDFT calculations, we use the accurate static xc kernel reported and used in Ref. 31. This kernel, which is based on a parametrization ${ }^{32}$ of the diffusion Monte Carlo (DMC) calculations reported in Ref. 33 for the uniform electron gas, was constructed for jellium surfaces where neglecting the $\omega$ dependence does not introduce significant errors and is expected to yield exact results in the limits of small and large wave vectors. Our numerical scheme was described in detail in Ref. 3, where only RPA calculations were reported.

The wave-vector-resolved exact-exchange surface energy $\gamma_{x}(k)$ is shown in Fig. 6. Figures 7-9 show, respectively, the wave-vector-resolved $\mathrm{xc}$ and correlation-only surface energies $\gamma_{\mathrm{xc}}(k)$ and $\gamma_{c}(k)$. Figure 6 shows that $\gamma_{x}^{\mathrm{PBEsol}}(k) \mathrm{im}-$ proves over PBE, as expected, and is close to the exact $\gamma_{x}(k)$ for intermediate values of the wave vector. At large values of the wave vector, both PBE and PBEsol correctly recover the nonoscillatory LSDA (see Figs. 1 and 2 of Ref. 3); differences between this nonoscillatory PBEsol (and also LSDA and PBE) and the exact $\gamma_{x}(k)$ at these large values of $k$ are due to the inaccuracy of the nonoscillatory model employed in Eq. (10) near $k=2 k_{F}$.

In Fig. 7, we compare the wave-vector-resolved exactRPA surface energy (as reported in Ref. 3) with its TDDFT

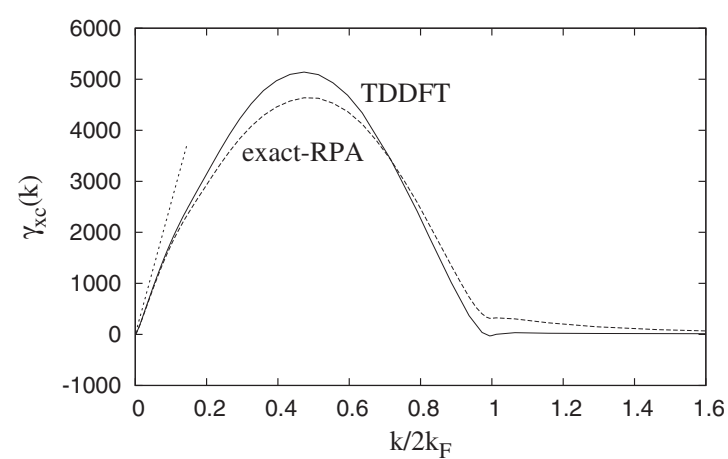

FIG. 7. Exact-RPA and benchmark TDDFT wave-vectorresolved xc surface energies $\gamma_{\mathrm{xc}}(k)$ versus $k / 2 k_{F}$ for a jellium slab of thickness $a=2.23 \lambda_{F}$ and $r_{s}=2.07$. The area under each curve represents the corresponding xc surface energy: $\sigma_{\mathrm{xc}}^{\mathrm{RPA}}$ $=3091 \mathrm{erg} / \mathrm{cm}^{2}$ and $\sigma_{\mathrm{xc}}^{\mathrm{TDDFT}}=3090 \mathrm{erg} / \mathrm{cm}^{2}$. The straight dotted line represents the universal low wave-vector limit of Eq. (19).

counterpart (which we have not reported elsewhere, and which required three months of computation). In the longwavelength limit $(k \rightarrow 0)$, both RPA and TDDFT calculations approach the exact low wave-vector limit of Eq. (19). In the large- $k$ limit, where RPA is wrong, our TDDFT approach (which reproduces accurately the xc energy of the uniform electron gas) is expected to be accurate. Furthermore, the uniform-gas-based isotropic xc kernel that we use in our TDDFT calculation has been shown recently to yield essentially the same two-dimensional (2D) wave-vector analysis as a more sophisticated high-level correlated approach (the inhomogeneous Singwi-Tosi-Land-Sjölander method) which does not use an isotropic kernel derived from the uniform gas. ${ }^{34}$ Hence, we take the TDDFT wave-vector-resolved surface energy represented in Fig. 7 by a solid line as the benchmark curve against which we compare various GGAs.

Figure 8 shows our wave-vector-resolved TDDFT surface energy together with its PBE and PBEsol counterparts. $\gamma_{\mathrm{xc}}^{\mathrm{PBEs}}$ is nearly exact at small wave vectors, where it

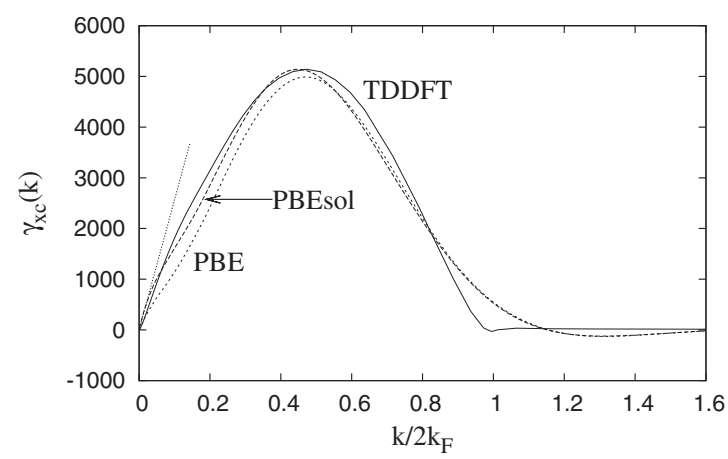

FIG. 8. PBE, PBEsol, and benchmark TDDFT wave-vectorresolved xc surface energies $\gamma_{\mathrm{xc}}(k)$ versus $k / 2 k_{F}$ for a jellium slab of thickness $a=2.23 \lambda_{F}$ and $r_{s}=2.07$. The semilocal PBE and PBEsol calculations have been performed from nonoscillatory parametrizations of the dimensionless exchange-hole shapes $J^{\mathrm{PBE}}$ and $J^{\mathrm{PBEsol}}$, respectively. The area under each curve represents the corresponding xc surface energy: $\sigma_{\mathrm{xc}}^{\mathrm{PBE}}=2885 \mathrm{erg} / \mathrm{cm}^{2}, \quad \sigma_{\mathrm{xc}}^{\mathrm{PBEs}}$ $=3027 \mathrm{erg} / \mathrm{cm}^{2}$, and $\sigma_{\mathrm{xc}}^{\mathrm{TDDFT}}=3090 \mathrm{erg} / \mathrm{cm}^{2}$. The straight dotted line represents the universal low wave-vector limit of Eq. (19). 


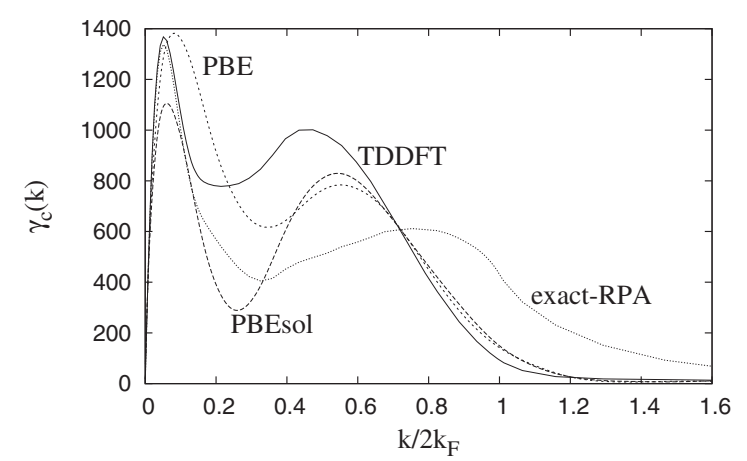

FIG. 9. PBE, PBEsol, exact-RPA, and benchmark TDDFT wave-vector-resolved correlation surface energies $\gamma_{c}(k)$ versus $k / 2 k_{F}$ for a jellium slab of thickness $a=2.23 \lambda_{F}$ and $r_{s}=2.07$. The area under each curve represents the corresponding correlation surface energy: $\sigma_{c}^{\mathrm{PBE}}=720 \mathrm{erg} / \mathrm{cm}^{2}, \quad \sigma_{c}^{\mathrm{PBEsol}}=604 \mathrm{erg} / \mathrm{cm}^{2}, \quad \sigma_{c}^{\mathrm{RPA}}$ $=743 \mathrm{erg} / \mathrm{cm}^{2}$, and $\sigma_{c}^{\mathrm{TDDFT}}=742 \mathrm{erg} / \mathrm{cm}^{2}$.

matches the exact initial slope of Eq. (19). At intermediate wave vectors, $\gamma_{\mathrm{xc}}^{\mathrm{PBEsol}}$ is very close to our benchmark TDDFT calculation. As in the case of wave-vector-resolved exchange-only surface energies, PBE and PBEsol calculations correctly recover the nonoscillatory LSDA and differ from the more accurate TDDFT calculation due to the inaccuracy of the nonoscillatory model of the exchange-hole shape [see, e.g., Eq. (10)]. Figure 8 shows that $\gamma_{\mathrm{xc}}^{\mathrm{PBEsol}}$ nicely matches our benchmark TDDFT calculation at low and intermediate wave vectors, so we recommend using the model PBEsol xc hole, in all solid-state calculations where the hole is needed but full nonlocality is not important, instead of the more expensive TDDFT. We recall that the system-averaged hole, unlike the energy density, is uniquely defined and is an observable at full coupling strength. ${ }^{5}$

Figure 9 exhibits the wave-vector-resolved correlationonly PBE, PBEsol, exact-RPA, and TDDFT surface energies.
We observe that at long wavelengths $(k \rightarrow 0)$, where the LSDA is known to fail badly, and at short wavelengths (large $k$ ), where RPA is wrong, the GGAs under consideration are considerably close to our benchmark TDDFT calculations. At intermediate wave vectors, however, GGAs cannot describe $\gamma_{c}$ accurately, although PBEsol has been shown in Fig. 8 to give a very good description of $\gamma_{\mathrm{xc}}$. This is due to a cancellation of the errors introduced at these wave vectors within the exchange and correlation contributions to $\gamma_{\mathrm{xc}}$, which almost cancel each other. ${ }^{35}$

\section{CONCLUSIONS}

We have constructed a PBEsol angle-averaged xc hole $\bar{n}_{\mathrm{xc}}(\mathbf{r}, u)$ that satisfies known exact constraints and recovers the recently reported PBEsol xc energy functional. Our construction of the PBEsol xc hole begins from and appropriately modifies the sharp cutoff correlation hole of Ref. 5 and the smooth exchange hole of Ref. 6. We also generalize [see Eq. (7)] the sharp cutoff procedure for the correlation hole to any GGA which has a positive gradient-expansion coefficient. We have found that our PBEsol xc hole describes accurately the wave-vector-resolved xc jellium surface energy for all values of the wave vector, thus providing support for the PBEsol GGA for solids and surfaces.

\section{ACKNOWLEDGMENTS}

L.A.C. and J.P.P. acknowledge NSF support (Grant No. DMR05-01588). J.M.P. acknowledges partial support by the Spanish MEC (Grant No. FIS2006-01343 and CSD2006-53) and the EC 6th framework Network of Excellence NANOQUANTA. L.A.C. thanks the Donostia International Physics Center (DIPC) where this work was started.
${ }^{1}$ W. Kohn and L. J. Sham, Phys. Rev. 140, A1133 (1965).

${ }^{2}$ D. C. Langreth and J. P. Perdew, Phys. Rev. B 15, 2884 (1977); 21, 5469 (1980); 26, 2810 (1982).

${ }^{3}$ J. M. Pitarke, L. A. Constantin, and J. P. Perdew, Phys. Rev. B 74, 045121 (2006).

${ }^{4}$ J. P. Perdew, K. Burke, and M. Ernzerhof, Phys. Rev. Lett. 77, 3865 (1996).

${ }^{5}$ J. P. Perdew, K. Burke, and Y. Wang, Phys. Rev. B 54, 16533 (1996); 57, 14999(E) (1998).

${ }^{6}$ M. Ernzerhof and J. P. Perdew, J. Chem. Phys. 109, 3313 (1998).

${ }^{7}$ L. A. Constantin, J. P. Perdew, and J. Tao, Phys. Rev. B 73, 205104 (2006).

${ }^{8}$ J. Tao, J. P. Perdew, V. N. Staroverov, and G. E. Scuseria, Phys. Rev. Lett. 91, 146401 (2003).

${ }^{9}$ J. P. Perdew, L. A. Constantin, E. Sagvolden, and K. Burke, Phys. Rev. Lett. 97, 223002 (2006).

${ }^{10}$ P. R. Antoniewicz and L. Kleinman, Phys. Rev. B 31, 6779 (1985).

${ }^{11}$ S.-K. Ma and K. A. Brueckner, Phys. Rev. 165, 18 (1968).
${ }^{12}$ J. P. Perdew, A. Ruzsinszky, G. I. Csonka, O. A. Vydrov, G. E. Scuseria, L. A. Constantin, X. Zhou, and K. Burke, Phys. Rev. Lett. 100, 136406 (2008); 102, 039902(E) (2009).

${ }^{13}$ R. Armiento and A. E. Mattsson, Phys. Rev. B 72, 085108 (2005).

${ }^{14}$ W. Kohn and A. E. Mattsson, Phys. Rev. Lett. 81, 3487 (1998).

${ }^{15}$ M. P. Johansson, A. Lechtken, D. Schooss, M. M. Kappes, and F. Furche, Phys. Rev. A 77, 053202 (2008).

${ }^{16}$ G. I. Csonka, A. Ruzsinszky, J. P. Perdew, and S. Grimme, J. Chem. Theory Comput. 4, 888 (2008).

${ }^{17}$ R. Wahl, D. Vogtenhuber, and G. Kresse, Phys. Rev. B 78, 104116 (2008)

${ }^{18}$ J. P. Perdew and Y. Wang, Phys. Rev. B 45, 13244 (1992).

${ }^{19}$ G. L. Oliver and J. P. Perdew, Phys. Rev. A 20, 397 (1979).

${ }^{20}$ J. P. Perdew and Y. Wang, Phys. Rev. B 46, 12947 (1992).

${ }^{21}$ T. M. Henderson, B. G. Janesko, and G. E. Scuseria, J. Chem. Phys. 128, 194105 (2008).

22 J. P. Perdew, J. Tao, V. N. Staroverov, and G. E. Scuseria, J. Chem. Phys. 120, 6898 (2004). 
${ }^{23}$ We expect that our xc hole will have practically the same accuracy as the PBEsol xc energy functional for the lattice-constant tests presented in Ref. 12 because the PBEsol exchange is very accurately fitted in Figs. 4 and 5. It was shown in the supporting information of Ref. 12 that the lattice constants are not sensitive to the details of the gradient dependence of the correlation functional (which is less well fitted in Figs. 4 and 5).

${ }^{24}$ This equation holds for a semi-infinite jellium system. For a jellium slab, the right-hand side of Eq. (17) should be divided by a factor of 2 .

${ }^{25}$ Z. Yan, J. P. Perdew, S. Kurth, C. Fiolhais, and L. Almeida, Phys. Rev. B 61, 2595 (2000).

${ }^{26}$ J. Harris and A. Griffin, Phys. Rev. B 11, 3669 (1975).

${ }^{27}$ O. Gunnarsson and B. I. Lundqvist, Phys. Rev. B 13, 4274 (1976).

${ }^{28}$ J. M. Pitarke and A. G. Eguiluz, Phys. Rev. B 57, 6329 (1998); 63, 045116 (2001).
${ }^{29}$ E. K. U. Gross, J. F. Dobson, and M. Petersilka, in Density Functional Theory II: Topics in Current Chemistry, edited by R. F. Nalewajski (Springer, Berlin, 1996), Vol. 181, p. 81.

${ }^{30}$ E. K. U. Gross and W. Kohn, Phys. Rev. Lett. 55, 2850 (1985).

${ }^{31}$ J. M. Pitarke and J. P. Perdew, Phys. Rev. B 67, 045101 (2003).

${ }^{32}$ M. Corradini, R. Del Sole, G. Onida, and M. Palummo, Phys. Rev. B 57, 14569 (1998).

${ }^{33}$ S. Moroni, D. M. Ceperley, and G. Senatore, Phys. Rev. Lett. 75, 689 (1995).

${ }^{34}$ L. A. Constantin, J. M. Pitarke, J. F. Dobson, A. García-Lekue, and J. P. Perdew, Phys. Rev. Lett. 100, 036401 (2008).

${ }^{35}$ Figures 6 and 9 show that for small wave vectors $\left(k / 2 k_{F}\right.$ $<0.05)$ and for $k / 2 k_{F}$ between 0.25 and $0.4, \gamma_{x}^{\mathrm{PBE}}(k)$ and $\gamma_{c}^{\mathrm{PBE}}(k)$ are more accurate than their PBEsol analogs. However, due to error cancellation between exchange and correlation, $\gamma_{\mathrm{xc}}^{\mathrm{PBEsol}}(k)$ is considerably closer to $\gamma_{\mathrm{xc}}^{\mathrm{TDDFT}}(k)$ than $\gamma_{\mathrm{xc}}^{\mathrm{PBE}}(k)$ for $k / 2 k_{F} \leq 0.5$ (see Fig. 8). 